\title{
Direct visualization of improved optic nerve pial vascular supply following tuberculum meningioma resection: case report
}

\author{
Seunggu J. Han, MD, ${ }^{1}$ Stephen T. Magill, MD, PhD, ${ }^{1}$ Phiroz E. Tarapore, MD, ${ }^{1}$ \\ Jonathan C. Horton, MD, PhD, ${ }^{2}$ and Michael W. McDermott, MD' \\ Departments of ${ }^{1}$ Neurological Surgery and ${ }^{2}$ Ophthalmology, University of California, San Francisco, California
}

Tuberculum sellae meningiomas frequently produce visual loss by direct compression from tumor, constriction of the optic nerve (ON) under the falciform ligament, and/or ON ischemia. The authors hypothesized that changes in visual function after tumor removal may be related to changes in blood supply to the $\mathrm{ON}$ that might be seen in the pial circulation at surgery. Indocyanine green (ICG) angiography was used to attempt to document these changes at surgery. The first patient in whom the technique was used had a left-sided, 1.4-cm, tuberculum meningioma. Time-lapse comparison of images was done postsurgery, and the comparison of video images revealed both faster initial filling and earlier complete filling of the ON pial circulation, suggesting improved pial blood flow after surgical decompression. In follow-up the patient had significant improvements in both visual acuity and visual fields function. Intraoperative ICG angiography of the $\mathrm{ON}$ can demonstrate measurable changes in pial vascular flow that may be predictive of postoperative visual outcome. The predictive value of this technique during neurosurgical procedures around the optic apparatus warrants further investigation in a larger cohort.

http://thejns.org/doi/abs/10.3171/2015.6.JNS15765

KEY WORDS indocyanine green; tuberculum sellae; meningioma; pial circulation; pial supply; optic nerve; oncology

$\mathrm{P}$ ATIENTS with meningiomas arising from the tuberculum sellae frequently present with visual loss. ${ }^{7,10}$ Accordingly, the main objective of treatment is preservation of vision, but tuberculum meningiomas remain challenging neurosurgical diseases to treat because visual loss is also one of the main risks of resection. ${ }^{9,11}$ The exact mechanism of visual loss in cases of growing mass lesions around the optic apparatus remains unclear, and direct compression, demyelination, and ischemia have been proposed as potential causes. ${ }^{7}$ However, it appears relatively common to observe compression, small-vessel compromise, and demyelination occurring concurrently. ${ }^{6}$

Vascular supply to the optic nerve (ON) does appear to play a significant role in both visual compromise on presentation and vision recovery on treatment and resection of the offending lesion. This concept is supported by reports of cases of worsening postoperative visual function in which imaging fails to reveal an operative site hematoma, subperiosteal orbital hematoma, or carotid/ophthal- mic artery vasospasm, and it is thought that the pial blood supply to the optic apparatus has been compromised..$^{9}$ To further explore the relationship between the vascular supply of the ON and visual outcomes in skull base meningioma surgery, the technique of intraoperative indocyanine green (ICG) angiography was used. Here, we report the use of ICG video angiography to evaluate the pial vascular supply of the ON compressed by a tuberculum meningioma, before and after tumor resection with decompression of the ON.

\section{Case Report}

History and Examination

A 52-year-old man presented with gradual blurring of vision in the left eye over 8 months. He was referred to an ophthalmologist who measured a best corrected visual acuity of 20/20 in the right eye and 20/30 in the left eye. A severe visual field defect affecting only the left eye was

ABBREVIATIONS $\mathrm{CPCON}=$ complete filling of the pial circulation of the optic nerve; ICA = internal carotid artery; ICG $=$ indocyanine green; iPCON $=$ initial filling of the pial circulation of the optic nerve; $\mathrm{ON}=$ optic nerve; $\mathrm{PCON}=$ pial circulation of the optic nerve.

SUBMITTED April 6, 2015. ACCEPTED June 17, 2015.

INCLUDE WHEN CITING Published online December 18, 2015; DOI: 10.3171/2015.6.JNS15765. 
detected on Humphrey visual field testing (Fig. 1). He had no other signs or symptoms; specifically he denied having headache, periorbital pain, or any facial numbness. His neurological examination was notable for temporal ON pallor in the left eye. He was otherwise neurologically intact with normal mental status, normal function of the 3rd-12th cranial nerves, full motor strength, intact sensation, normal reflexes, and normal gait.

Hematological and electrolyte laboratory examinations showed no abnormal results. Magnetic resonance imaging of the brain with and without contrast revealed a $14 \times 9.4 \times 9.7-\mathrm{mm}$ homogeneously enhancing mass medial to the anterior clinoid process on the left involving the optic canal, which was most consistent with a tuberculum sellae meningioma (Fig. 2). There was no evidence of involvement of the left cavernous sinus, or extension of the mass into the orbital apex. Treatment options for the lesion including open surgical, endoscopic, and radiotherapy options were discussed with the patient, and the decision was made to proceed with resection with decompression of the $\mathrm{ON}$.

\section{Operation}

The use of ICG to study the pial circulation of the ON (PCON) in meningioma surgery was approved by the local Committee on Human Research, and the patient provided consent for its use before and after tumor resection. The patient underwent a left frontotemporal craniotomy with orbital osteotomy for tumor resection. Following the orbital osteotomy the optic canal was opened extradurally

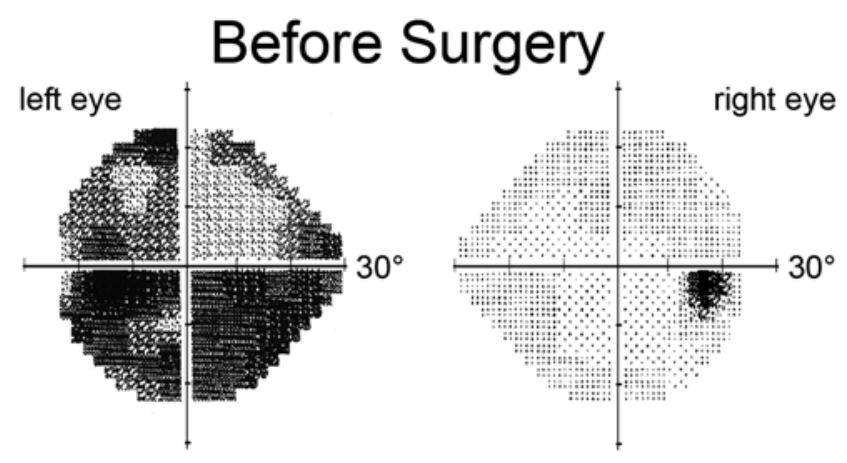

After Surgery
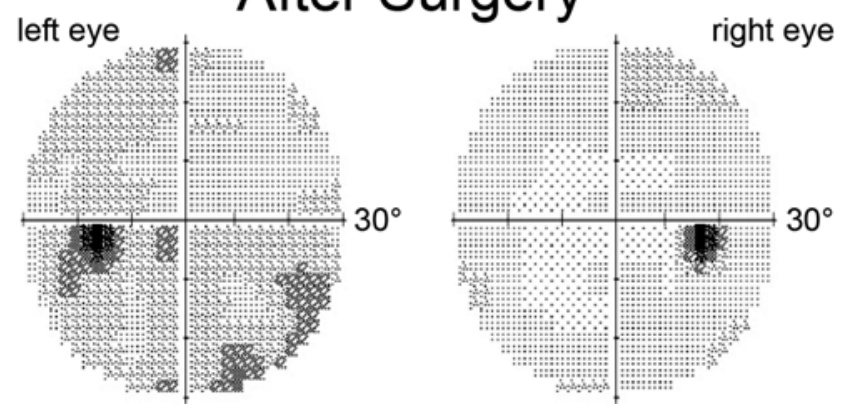

FIG. 1. Upper row: Visual field examinations performed on a Humphrey perimeter show severe depression of retinal sensitivity in the left eye before surgery, with a mean deviation of $-16 \mathrm{~dB}$. Lower row: After surgery the visual field has returned to nearly normal, with a mean deviation of only $-5 \mathrm{~dB}$.
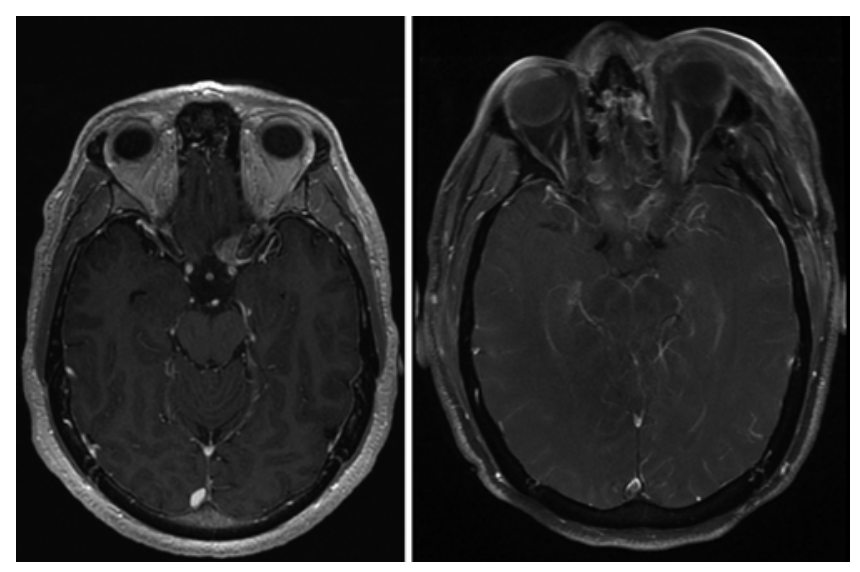

FIG. 2. Contrast-enhanced T1-weighted MRI study of the brain showing a 1.4-cm left tuberculum sellae meningioma (left). Fat-suppressed contrast-enhanced T1-weighted MRI study performed postoperatively that shows radiographic gross-total resection (right).

without clinoidectomy. On intradural examination, the $\mathrm{ON}$ was found to be displaced superiorly and laterally by a tumor arising from the junction of the chiasmatic sulcus and proximal medial left optic canal (Fig. 3 left). A Simpson Grade II removal of a left tuberculum meningioma was accomplished without complications ${ }^{13}$ (Fig. 3 right).

\section{Analysis of PCON by ICG Angiography}

During the operation, ICG was injected intravenously, and intraoperative recordings of the PCON were made using a microscope with dedicated software (Pentero, Carl Zeiss Co.). The ICG angiography studies were performed at two points during the procedure: once before and then again after resection of the tumor (Video 1).

VIDEO 1. Indocyanine green video angiography performed at two points during the procedure: once before and then again after resection of the tumor. The intervals between the first appearance of ICG in the ICA and IPCON and between IPCON and CPCON were marked and measured at both pre- and postresection states. After resection, there is both faster iPCON and earlier CPCON, suggesting improved pial blood flow after surgical decompression. Copyright Seunggu J. Han. Published with permission. Click here to view.

The intervals between 1) first appearance of ICG in the internal carotid artery (ICA) to initial filling of the pial circulation of the ON (iPCON) and 2) iPCON to complete filling of the PCON (cPCON) were recorded and measured at both time points. Qualitative review of pre- and postresection ICG angiograms at the time of surgery revealed a shorter ICA-to-iPCON time and shorter iPCON-to-cPCON times after tumor removal. Preresection to postresection ICA-toiPCON times were 4.1 and 2.4 seconds, respectively. Preresection to postresection iPCON-to-cPCON times were 12.2 and 8.4 seconds, respectively.

Quantitative ICG signal intensity analysis was also performed to confirm these impressions. From the original ICG video, still images were extracted for analysis at 1 -second intervals. From these images, the ICG signal intensities of the ICA and the ON were quantified using ImageJ software (NIH). The reference start time was determined when the ICA had a relative signal intensity of 100 . 

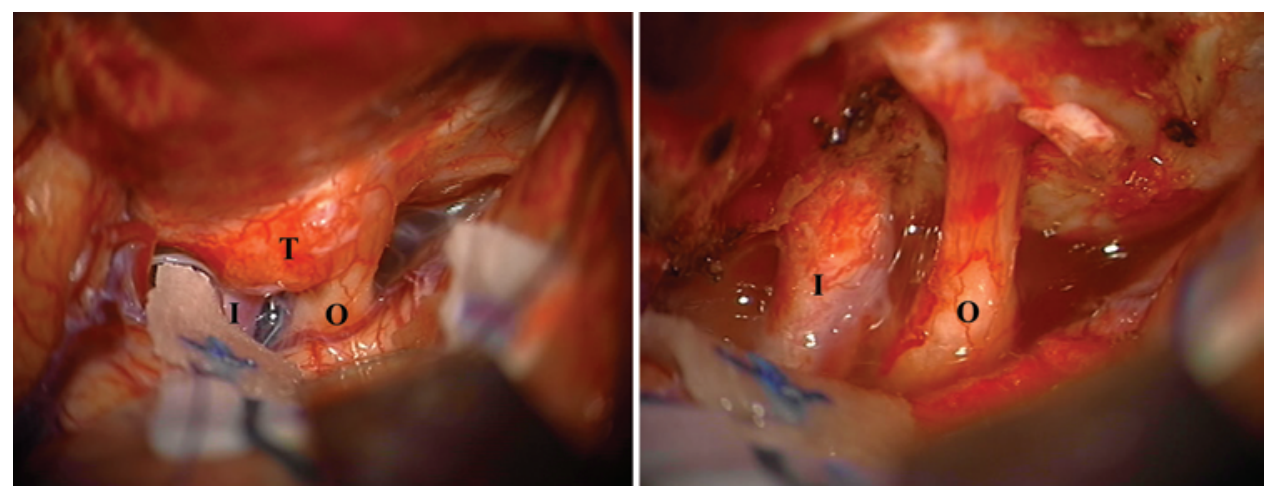

FIG. 3. Intraoperative images of tumor resection performed via a left frontotemporal craniotomy with orbital osteotomy approach. Left: Preresection view showing superior and lateral displacement of the left ON by tumor arising from the junction of the chiasmatic sulcus and proximal medial left optic canal. Right: Postresection images showing good decompression of the ON after Simpson Grade II resection. I = ICA; O = ON; $\mathrm{T}$ = tumor. Figure is available in color online only.

Referencing back to the original images, the signal intensity threshold corresponding to the iPCON time point was found to be 80 , and this threshold was confirmed to be reached by the $\mathrm{ON}$ in the preresection state at 4 seconds, and by the $\mathrm{ON}$ on the postresection images between 2 and 3 seconds (Figs. 4 and 5). The cPCON time point corresponded to a signal intensity value of 200 , which was reached after 12 seconds in the preresection $\mathrm{ON}$, and between 8 and 9 seconds in the postresection ON.

\section{Postoperative Course}

The patient tolerated the procedure well and suffered no new neurological symptoms. He described his vision as stable postoperatively compared with before tumor resection. He was discharged home in good condition on postoperative Day 4. Repeat MRI performed postoperatively revealed radiographic gross-total resection (Fig. 2 right).

In outpatient follow-up, 2 weeks postprocedure, the patient had improvements in subjective visual acuity and

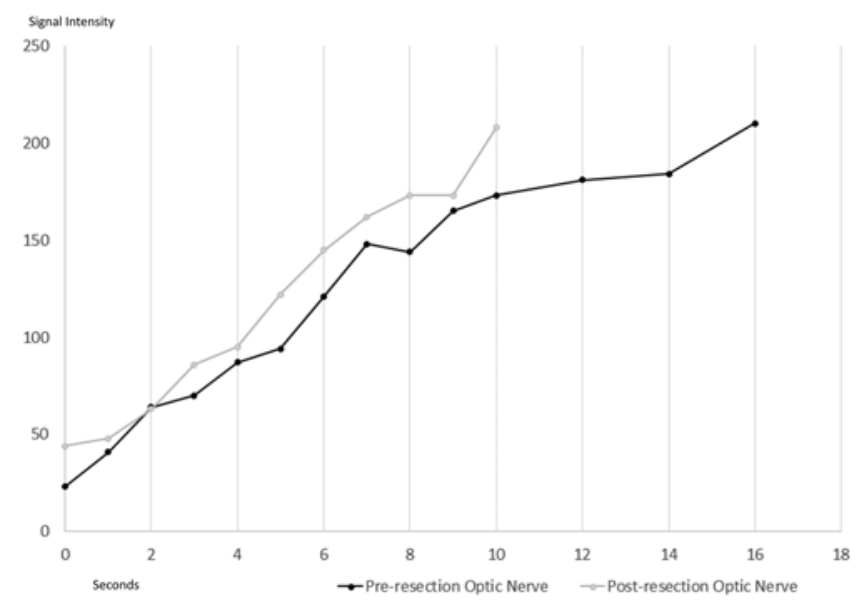

FIG. 4. Chart showing intensity of the ICG signal in the ON; ICG signal intensity of the $\mathrm{ON}$ in preresection and postresection states as calculated by Image $\mathrm{J}$ over time. Time 0 is defined as the ICA reaching an intensity of 100 . There is an earlier rise of the ICG signal intensity in the ON after tumor removal. color perception. Testing by an ophthalmologist 6 months after surgery documented a visual acuity of 20/20 in each eye. The visual field defect had resolved nearly completely (Fig. 1). The patient is currently being followed with serial imaging, and remains recurrence free 4 years postresection.

\section{Histopathological Findings}

Microscopic evaluation of the resected tumor tissue and involved clinoid dura mater revealed WHO Grade I meningioma, without evidence of high-grade features.

\section{Discussion}

Intraoperative video angiography performed using ICG for visualization and recording through operating microscopes has been widely embraced by neurosurgeons, often replacing intraoperative conventional digital subtraction angiography in the management of intracranial aneurysms. ${ }^{1,412}$ In addition, ICG angiography has the benefit of greater detail and direct visualization and the ability to covisualize nonvascular anatomical structures with light microscopy. The indications for ICG angiography have expanded since its introduction into the neurosurgical arena to include arteriovenous malformation, ${ }^{16}$ intracranial revascularization, ${ }^{1}$ spinal dural arteriovenous fistula, ${ }^{2}$ intraaxial tumor resection, ${ }^{3}$ endoscopic third ventriculostomy ${ }_{15}$ and microvascular decompression. ${ }^{17}$ The use of ICG in meningioma surgery has been described, demonstrating its potential benefit in identifying adjacent venous sinuses and pial supply of the tumors. ${ }^{14}$ We report the first application of ICG to study the pial circulation of the ON during meningioma surgery around the optic apparatus.

In our case, the ICG allowed us to visualize improved pial vascular supply to the affected ON, with faster iPCON and earlier cPCON after tumor resection. Fortunately, the patient experienced significant recovery in visual function over time after surgery. Recognizing vision preservation as a top priority in surgical management of tuberculum meningiomas, previous reports have described different preoperative variables predictive of improved visual outcomes after treatment. ${ }^{5,8}$ These variables include dura- 

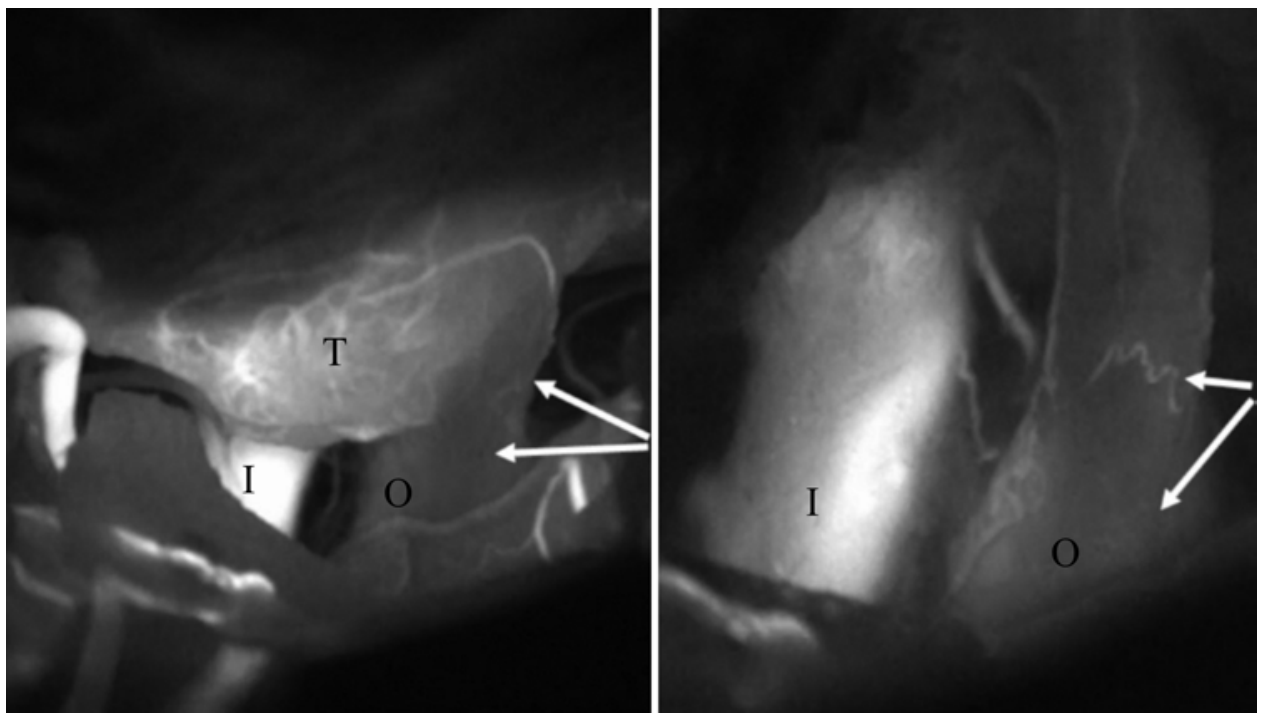

FIG. 5. Indocyanine green video angiography showing earlier filling of pial vasculature (arrows) of the ON after decompression and tumor removal. Both images were taken 5 seconds after filling of the ICA to an intensity value of 100 . Left: Filling prior to tumor resection. Right: Filling after tumor resection.

tion of symptoms, intrinsic signal characteristics on MRI, presence of peritumoral edema, extent of resection, and involvement of surrounding vessels. We believe that the improvement of PCON seen on ICG angiography may also be of prognostic value for early improvement of visual function. Although the technique certainly warrants examination across a larger cohort, one of its strengths is that the filling times of the pial circulation relative to the ICA can be measured and tracked in an objective fashion. In addition, our findings continue to support the role of local vascular supply in visual outcomes for patients undergoing treatment for tuberculum meningioma as well as other compressive mass lesions around the optic apparatus.

\section{Conclusions}

We report the first case in which ICG angiography has been applied to measure the PCON during resection of a tuberculum meningioma compressing the left $\mathrm{ON}$. This technique appears to be feasible and may have a role in predicting early visual outcomes in patients undergoing resection of mass lesions around the optic apparatus.

\section{References}

1. Abla AA, Lawton MT: Indocyanine green angiography for cerebral aneurysm surgery: advantages, limitations, and neurosurgeon intuition. World Neurosurg 82:e585-e586, 2014

2. Desai A, Bekelis K, Erkmen K: Minimally invasive tubular retractor system for adequate exposure during surgical obliteration of spinal dural arteriovenous fistulas with the aid of indocyanine green intraoperative angiography. J Neurosurg Spine 17:160-163, 2012

3. Ferroli P, Acerbi F, Albanese E, Tringali G, Broggi M, Franzini A, et al: Application of intraoperative indocyanine green angiography for CNS tumors: results on the first 100 cases. Acta Neurochir Suppl 109:251-257, 2011

4. Hardesty DA, Thind H, Zabramski JM, Spetzler RF, Nakaji P: Safety, efficacy, and cost of intraoperative indocyanine green angiography compared to intraoperative catheter angiography in cerebral aneurysm surgery. J Clin Neurosci 21:1377-1382, 2014

5. Kim TW, Jung S, Jung TY, Kim IY, Kang SS, Kim SH: Prognostic factors of postoperative visual outcomes in tuberculum sellae meningioma. Br J Neurosurg 22:231-234, 2008

6. Lee JH, Jeun SS, Evans J, Kosmorsky G: Surgical management of clinoidal meningiomas. Neurosurgery 48:10121021, 2001

7. Mahmoud M, Nader R, Al-Mefty O: Optic canal involvement in tuberculum sellae meningiomas: influence on approach, recurrence, and visual recovery. Neurosurgery 67 (3 Suppl Operative):ons108-ons119, 2010

8. Palani A, Panigrahi MK, Purohit AK: Tuberculum sellae meningiomas: A series of 41 cases; surgical and ophthalmological outcomes with proposal of a new prognostic scoring system. J Neurosci Rural Pract 3:286-293, 2012

9. Santarius T, Jian BJ, Englot D, McDermott MW: Delayed neurological deficit following resection of tuberculum sellae meningioma: report of two cases, one with permanent and one with reversible visual impairment. Acta Neurochir (Wien) 156:1099-1102, 2014

10. Schick U, Hassler W: Surgical management of tuberculum sellae meningiomas: involvement of the optic canal and visual outcome. J Neurol Neurosurg Psychiatry 76:977-983, 2005

11. Seol HJ, Park HY, Nam DH, Kong DS, Lee JI, Kim JH, et al: Clinical outcomes of tuberculum sellae meningiomas focusing on reversibility of postoperative visual function. Acta Neurochir (Wien) 155:25-31, 2013

12. Sharma M, Ambekar S, Ahmed O, Nixon M, Sharma A, Nanda A, et al: The utility and limitations of intraoperative near-infrared indocyanine green videoangiography in aneurysm surgery. World Neurosurg 82:e607-e613, 2014

13. Simpson D: The recurrence of intracranial meningiomas after surgical treatment. J Neurol Neurosurg Psychiatry 20:22-39, 1957

14. Ueba T, Okawa M, Abe H, Nonaka M, Iwaasa M, Higashi T, et al: Identification of venous sinus, tumor location, and pial supply during meningioma surgery by transdural indocyanine green videography. J Neurosurg 118:632-636, 2013

15. Wachter D, Behm T, von Eckardstein K, Rohde V: Indocya- 
nine green angiography in endoscopic third ventriculostomy. Neurosurgery 73 (1 Suppl Operative):ons67-ons73, 2013

16. Zaidi HA, Abla AA, Nakaji P, Chowdhry SA, Albuquerque FC, Spetzler RF: Indocyanine green angiography in the surgical management of cerebral arteriovenous malformations: lessons learned in 130 consecutive cases. Neurosurgery 10 (Suppl 2):246-251, 2014

17. Zhang $\mathrm{H}, \mathrm{Fu} \mathrm{WM}$, Chen $\mathrm{P}$, Shi J: Intraoperative indocyanine green angiography during microvascular decompression surgery: report of 30 cases. Acta Neurochir (Wien) 156:15611564,2014

\section{Disclosures}

The authors report no conflict of interest concerning the materials of methods used in this study or the findings specified in this paper.

\section{Author Contributions}

Conception and design: McDermott, Han. Acquisition of data: Han, Magill, Tarapore. Analysis and interpretation of data:

McDermott, Han, Magill, Horton. Drafting the article: McDermott, Han, Magill, Horton. Critically revising the article: McDermott, Magill, Tarapore, Horton. Reviewed submitted version of manuscript: all authors.

\section{Supplemental Information \\ Videos}

Video 1. https://vimeo.com/146107570.

\section{Correspondence}

Michael W. McDermott, Department of Neurological Surgery, University of California, San Francisco, 505 Parnassus Ave., Box 0112, San Francisco, CA 94143. email: mcdermottm@neurosurg. ucsf.edu. 